\title{
Study of Riveting Machine Riveting Device Based on Conceptual Design
}

\author{
Jiaqing Jian ${ }^{* 1, a}$, Guoquan Zhang, ${ }^{1, b}$, Hongwei Fang ${ }^{1, d}$ \\ ${ }^{1}$ School of Mechanical Engineering, Wuhan polytechnic \\ University, Wuhan, P.R.China \\ aJianjia620@126.com, ${ }^{\mathrm{b}} 531369886 @ q q . c o m$, \\ d824470338@qq.com
}

\begin{abstract}
In order to improve the automation of capacitor package, conceptual design and fuzzy theory is applied to establish evaluation system of the riveting device and evaluation index system of hierarchy analysis, resulting in a variety of design schemes, and finally, through the individual level relative membership degree matrix and integrated system solution, get the best program. The assessment method adopted, in this paper, conforms to the features of conceptual design, and has a good application prospect.
\end{abstract}

Keywords-conceptual design; project evaluation; riveting device; fuzzy set theory analysis unit

\section{INTRODUCTION}

At present, encapsulated in the capacitor industry still mainly manual packing, due to the capacitor encapsulation has certain corrosion and because the artificial cost is increasing, so from the manual or semi-automatic operation to highly automated development is an inevitable trend[1].Capacitor cover plate is an important part of a capacitor, riveting was carried out on the cover plate is the key step of capacitor encapsulation. Riveting device is to cover and cover capacitors with riveted together, so that the connections are secure.

Currently: conceptual design, there are two relatively authoritative definitions: G Pahl and W Beitz in his book, the Engineering Design of the conceptual design is defined as: "after the task of determining, through abstraction, developing functional structure, finding the right combination of principle and determined the basic solution, that solution, this part of the design work is called the conceptual design."[2]M J Frence in his Conceptual Design for Engineers, the conceptual design is defined as: "the content of the conceptual design is to consider design issues and in the form of scheme put forward numerous solutions during the design phase."[3]And the design of concept is an important part of the most creative in the development process.

\section{The Principle Of The Riveting Device}

Riveting device is one of the most critical components in the capacitor equipment, foil and cover the riveting quality plays an important role. Basic design requirements are: riveting cover plates to be the accurate and reliable, easy installation and maintenance, riveting time is short, simple structure. Therefore, the structure shown in Figure

\author{
Zhiming $\mathrm{Yu}^{2, \mathrm{c}}$ \\ ${ }^{2}$ Full Avic Hubei Ali-JiaTai Aircraft Equipment Co.,LTD, \\ Xiangyang, P.R.China \\ c421984026@qq.com
}

1 below, its key component consists of upper and lower cams, riveted on the swing arm and drop spindles. Its work principle for: motor will power through shaft part passed to cam 1,led its rotating movement; thus will cam of rotating movement conversion for pendulum arm of upper and lower movement, and promoted bottom pressure riveting pendulum arm 4 of left end down movement, due to pendulum arm Central is fixed of, so pendulum arm right end is up movement; will movement passed to bottom sliding axis upper, and promoted bottom sliding axis up movement, thus makes bottom plate 6 also up movement; while; power also passed to sprocket 2,and through transition sprocket 9 will power passed to top sprocket; due to cam 2 and sprocket with axis, so will cam 2 of rotating movement becomes pendulum arm of upper and lower movement, thus makes top pressure riveting pendulum arm left end up movement, its right end is down movement; so makes upper sliding axis also down movement, thus makes upper plate 6 also down action, through upper and lower plate of while action will guide foil article and cover board riveted in together. Finally, each part back into place, waiting for the next cycle.

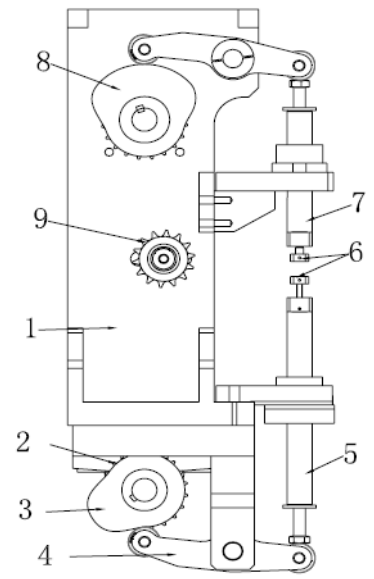

1-rivet base;2-sprocket;3-cam1;4-riveted swinging arm;5-slide shaft; 6-plate;7-sliding axle;8-cam2;9-transition-cam sprocket Figure 1 . The principle diagram of the plan 


\section{The Schemes Of The Riveted DeVICE EVALUATION SYSTEM}

For riveting equipment evaluation results as accurate as possible, to be effective, we must establish an evaluation index system. Conceptual design is a design process and evaluation throughout the process. Information in this process is gradually increasing and lucid, which with the conceptual design of the index system of the evaluation of the procedure should be changed. Evaluation index system of structure should satisfy two requirements: one is the evaluation indicator system should be as comprehensive as possible, but must get the point; the second is the evaluation system to constantly improve depending on design activities appropriate indicators. Therefore, the hierarchical structure model is used to establish evaluation index system of the mechanical system. There are several basic steps follows:(1)determining the index wight;(2) determining the relative membership degree matrix;(3)solving the basic unit system;(4)solving the comprehensive unit system.

\section{Project Evaluation Of The Riveting DEVICE FUNDAMENTALS}

In the process of conceptual design scheme evaluation, there is no absolute, or is a fuzzy concept.

The scheme evaluation and selection problem is one and a half structural system selection problem, that given a group of scheme $x_{1}, x_{2}, \ldots, x_{\mathrm{n}}$ formed scheme set $\mathrm{X}=\left\{x_{1}, x_{2}, \ldots, x_{\mathrm{n}}\right\}$, stay seeking of basic unit system has $\mathrm{m}$ a indicators, formed indicators set $\mathrm{Y}=\left\{d_{1}, d_{2}, \ldots, d_{n}\right\}$,participate in assessment of expert has $\mathrm{r}, \mathrm{Z}=\left\{x_{1}, x_{2}, \ldots, x_{\mathrm{r}}\right\}$, eventually decision of nature is optimization of process. Can be turned into a series of unit system selection problem to solve by half structural system. This evaluation system can be decomposed into a series of interdependent and interrelated systems in figure 2.The features of unit system: input is often a fuzzy matrix and the output matrix as fuzzy line[7].Solving the basic idea is to start at the bottom of the base unit system began, from low-level to high-level, and up to the highest level, namely the output layer. Every base unit system chosen is the relative membership degree matrix and the weight of the system, output is the relative membership degree vector, that is relative membership degree. When the integrated system solution is completed, can be conducted with the relative membership degree vector, that is the superiority of the sort of the program to select the optimal solution.
Comprehensive unit system $\mathrm{B}_{1} \mathrm{~B}_{2} \mathrm{~B}_{3} \mathrm{~B}_{4}$

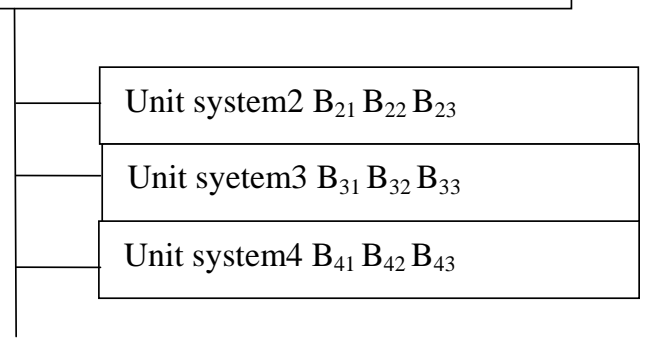

Figure 2. The system diagram

With conceptual design and fuzzy evaluation [6]get four different designs:(1)the cam driver and swing arm(2) the cam driver and connecting rod(3) Cylinder driver and deliver way swing arm(4) the cylinder driving and connecting rod transmission way.

\section{A. Determine the weight of evaluation indexes}

Fuzzy set analysis theory[4,5]is one of the basic methods in fuzzy learning, is also used in scheme evaluation. Solved the problem of indicators are not accurate in conceptual design, simple and practical.

In basic unit system 1 to illustrate the determination of index weight method, set index $\mathrm{D}=\left\{\mathrm{d}_{1}, \mathrm{~d}_{2}, \mathrm{~d}_{3}, \mathrm{~d}_{4}\right\}=\{$ reasonable structure, the manufacturing cost, reliability, maintenance convenience $\}$

\section{B. Qualitative sorting}

With 3 experts in scheme evaluation, according to their experience and knowledge of experts, the units important of system indicators binary compare, binary comparison matrix can be obtained.

Following three experts judgment available scale matrix of important are:

$$
\begin{aligned}
& \begin{array}{lllll}
d_{1} & d_{2} & d_{3} & d_{4}
\end{array} \\
& d_{1}\left[\begin{array}{llll}
0.5 & 1 & 0.5 & 1
\end{array}\right] \text { (3) } \\
& d_{2}\left[\begin{array}{lllll}
0 & 0.5 & 0.5 & 0.5 & (1.5)
\end{array}\right. \\
& \begin{array}{llllll}
d_{3} & 0.5 & 0.5 & 0.5 & 0.5 & \text { (2) }
\end{array} \\
& d_{4_{1}}\left[\begin{array}{llll}
0 & 0.5 & 0.5 & 0.5
\end{array}\right](1.5) \\
& \begin{array}{llll}
d_{1} & d_{2} & d_{3} & d_{4}
\end{array} \\
& d_{1}\left[\begin{array}{llll}
0.5 & 0.5 & 1 & 1
\end{array}\right] \text { (3) } \\
& \begin{array}{llllll}
d_{2} & 0.5 & 0.5 & 0.5 & 1 & (2.5)
\end{array} \\
& \begin{array}{lllllll}
d_{3} & 0 & 0.5 & 0.5 & 0 & \text { (1) }
\end{array} \\
& d_{4_{2}}\left[\begin{array}{llll}
0 & 0 & 1 & 0.5
\end{array}\right](1.5)
\end{aligned}
$$




$$
\begin{aligned}
& \begin{array}{llll}
d_{1} & d_{2} & d_{3} & d_{4}
\end{array} \\
& \begin{array}{l}
d_{1} \\
d_{2} \\
d_{3} \\
d_{43}
\end{array}\left[\begin{array}{cccc}
0.5 & 1 & 1 & 1 \\
0 & 0.5 & 0 & 1 \\
0 & 1 & 0.5 & 0.5 \\
0 & 0 & 0.5 & 0.5
\end{array}\right]\left(\begin{array}{c}
(3.5) \\
(1.5) \\
(1)
\end{array}\right.
\end{aligned}
$$

Among them, and on the right side of the figures in parentheses are per line.

By the above 3 matrix of 3 groups of lines can be obtained 3 experts on the available indicators set for comprehensive scale matrix of importance.

$$
F=\left[\begin{array}{cccc}
3 & 1.5 & 2 & 1.5 \\
3 & 2.5 & 1 & 1.5 \\
3.5 & 1.5 & 2 & 1
\end{array}\right]
$$

Familiarity with the set 3 experts in his own field: $O=\left(O_{1}, O_{2}, O_{3}\right)=(95,85,75)$,the normalized: obtained the weight of 3 experts $W^{2}=(0.373,0.333,0,294)$,be able to get 3 experts to the overall importance index set $\mathrm{D}$ consistency matrix:

$$
\mathrm{F}=\left[\begin{array}{lll}
0.373, & 0.333 & 0.294
\end{array}\right]\left[\begin{array}{cccc}
3 & 1.5 & 2 & 1.5 \\
3 & 2.5 & 1 & 1.5 \\
3.5 & 1.5 & 2 & 1
\end{array}\right]=
$$

Basic unit index ranking of the importance of the system are: $d_{1}, d_{2}, d_{3}, d_{4}$. Similarly, you can determine the sort of qualitative indicators in other unit systems.

\section{Qualitative ranking}

Indicators from unit on the basis of qualitative sorting, the most important indicators is, each expert compared the importance of qualitative indexes and other indicators, obtained the relative membership degree. According to reference[4]draw weight formula:

$$
w_{i}=\frac{1-g_{1 i}}{g_{1 i}} / \sum_{i=1}^{m} \frac{1-g_{1 i}}{g_{1 i}}, 0.5 \leq g_{1 i} \leq 1_{, \mathrm{i}=1,2, \ldots, \mathrm{m}}
$$

In binary quantitative comparison conforms to the language habits, Reference[5]voice operator is given, qualitative scale, the relationship between the relative membership degree, give through $g_{1 \mathrm{i}}$ the modal operator Judgment, can be set on the importance of relative membership degree vector,

$$
w^{\prime}=\left(w_{1}^{\prime}, w_{2}^{\prime}, \ldots w_{n}^{\prime}\right) .
$$

Set each expert weight and qualitative evaluation of the same value, have not normalized vector:

$$
\begin{aligned}
& W^{\prime}=\left(w_{1}^{2}, w_{2}^{2}, \ldots, w_{i}^{2}\right)=\left[\begin{array}{llll}
1_{w 1} & 1_{w 2} & 1_{w 3} & 1_{w 4} \\
2_{w 1} & 2_{w 2} & 2_{w 3} & 2_{w 4} \\
3 & 3 & 3
\end{array}\right]=\left[\begin{array}{lll}
0.373, & 0.333, & 0.294
\end{array}\right]\left[\begin{array}{cccc}
1 & 0.818 & 0.667 & 0.429 \\
1 & 0.818 & 0.538 & 0.42 \\
1 & 0.818 & 0.667 & 0.33
\end{array}\right] \\
& =\left[\begin{array}{llll}
1, & 0.818, & 0.624, & 0.4
\end{array}\right]
\end{aligned}
$$

Normalized basic unit system 1 weight of each indicator: $\mathrm{W}=(0.352,0.288,0.22,0.14)$

\section{Determine the relative membership degree matrix}

With basic unit system 1 as an example to illustrate the determination of the relative membership degree matrix. Through qualitative and quantitative methods to determine the unit 1 sorting system the relative membership degree vector[8].By binary comparison, sets can be obtained on the refinement of the indicators $d_{11}$. $d_{12}, d_{13}, d_{14}$ and respectively the relative membership degree vector:

$$
\begin{gathered}
d_{11}\left(r_{x 1}, r_{x 2}, r_{x 3}, r_{x 4}\right)=(1,1,0.538,1) \\
d_{12}\left(r_{x 1}, r_{x 2}, r_{x 3}, r_{x 4}\right)=(1,1,0.538,0.538) \\
d_{13}\left(r_{x 1}, r_{x 2}, r_{x 3}, r_{x 4}\right)=(1,0.538,1,0.538) \\
d_{14}\left(r_{x 1}, r_{x 2}, r_{x 3}, r_{x 4}\right)=(1,1,1,0.538)
\end{gathered}
$$

Set to the refinement of the indicators $d_{11}, d_{12}, d_{13}, d_{14}$ and relative membership degree matrix:

$$
d_{1} R=\left[\begin{array}{cccc}
1 & 1 & 0.538 & 1 \\
1 & 1 & 0.538 & 0.538 \\
1 & 0.538 & 1 & 0.538 \\
1 & 1 & 1 & 0.538
\end{array}\right]
$$

Based on relative membership degree of optimization model

$$
u_{j}=\frac{1}{1+\left[1+\frac{4}{\sum_{i=1}^{4} r_{i j}}\right]^{2}}
$$

Based on the above model can be get indicators d1of the relative membership degree vector

$$
d_{1}{ }^{r}=(0.272,0.267,0.249,0.212)
$$

Similarly can also get indicators $d_{2}, d_{3}, d_{4}$ of unit system 1 and the relative membership degree vector:

$$
\begin{aligned}
& d_{2}{ }^{r}=(0.269,0.263,0.246,0.222) \\
& d_{3}{ }^{r}=(0.299,0.292,0.178,0.23) \\
& d_{4} r=(0.261,0.272,0.225,0.243)
\end{aligned}
$$

So, get basic unit system 1 of relative membership degree matrix:

$$
{ }_{1} R=\left[\begin{array}{cccc}
0.272 & 0.267 & 0.249 & 0.212 \\
0.269 & 0.263 & 0.246 & 0.222 \\
0.299 & 0.292 & 0.178 & 0.23 \\
0.261 & 0.272 & 0.255 & 0.243
\end{array}\right]
$$




\section{E. Solved the basic unit system}

Program set belonging to various indicators of the relative membership degree matrix[8] $U=u_{h j}, u_{h j}$ for the program belong to the relative membership degree, satisfy the condition, $0 \leqslant u_{h j} \leqslant 1, \nabla j, \nabla h$ 。

According to reference[4] ,a multilevel fuzzy optimization evaluation model is established

$$
u_{h j}= \begin{cases}0, & d_{k j}=0, k \neq h \\ \sum_{k=1}^{c} \frac{\sum_{i}^{m}\left[w_{i}\left(r_{i j}-s_{h}\right)\right]^{2}}{\sum_{i}^{m}\left[w_{i}\left(r_{i j}-s_{k}\right)\right]^{2}}, & d_{k j} \neq 0, \forall i, j \\ 1, \quad \sum_{h=1}^{c} u_{h j}=1 & d_{h j}=0, h=k\end{cases}
$$

$u_{h j}$ indicates that the program $x_{j}$ on level $h$ membership levels, $s_{h}$ level $h$ characteristic values of relative membership degree. $d_{k j}$ indicates that the difference between scheme $j$ and level $k$,

$$
d_{k j}=\left\{\sum_{i=1}^{m}\left[w_{i}\left(r_{i j}-s_{h}\right)^{2}\right]\right\}^{1 / 2}
$$

A case study of basic unit system 1 , according to the index weight ${ }_{1} W=(0.352,0.288,0.22,0.14)$ as well as relative membership degree matrix ${ }_{1} R$, and according to the model can get the 4 program on the poor, middle and excellent membership matrix

$$
U=\left[\begin{array}{cccc}
0 & 0 & 0 & 0 \\
0.013 & 0.0014 & 0.1269 & 0.2462 \\
0.9987 & 0.9986 & 0.8731 & 0.7538
\end{array}\right]
$$

Characteristic values can be obtained: $\mathrm{H}=(1,2,3) \mathrm{U}=(2.999,2.999,2.873,2.754)$, got the members -hip degree of unit system 1(0.9996,0.9995,0.9577,0.9179).

\section{F. Solved the comprehensive unit system}

When the basic unit system is completed, it can be solved with a layer comprehensive unit system,

which can consist of a base unit system for 1-4 their relative membership degree vector output as input, according to the model can get 4 different schemes, and excellent in relative membership

degree matrix

$$
U=\left[\begin{array}{cccc}
0 & 0 & 0 & 0 \\
0.0022 & 0.0025 & 0.0188 & 0.0295 \\
0.9998 & 0.9975 & 0.9813 & 0.9705
\end{array}\right]
$$

obtained the characteristic value $H=(1,2,3) U=(3.0038$, $2.9975,2.9815,2.9705)$. According to the eigenvalue 4 set of scenarios to determine a ranking
as1,2,3,4.Consequently,program the cam driver and swing-arm drive mode best properties. Therefore, riveting device key components of the mechanism as shown in figure(3):

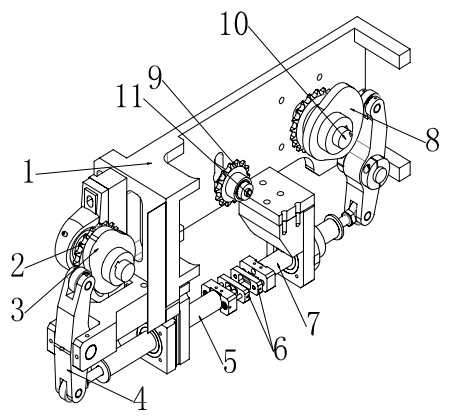

1-rivet base;2-sprocket;3-cam1;4-riveted swinging arm;5-slide shaft;6-plate;

7-sliding axle;8-cam2;9-transition-cam sprocket;10-shaft;11-idler shaft Figure 3. Structure Diagram

\section{V.CONCLUSION}

Based on the fuzzy set analysis theory, hierarchy of design index, and refined its structure for the 16-station according to design requirements, thereby changed the chaff to external disc and used the up and down movements to press simultaneously riveted two axis cover plate. The expert's experience, knowledge and objective data is utilized by the evaluation method in this paper. And consistent with the evaluation of conceptual design feature, it's a very effective method and makes the design process more rational, provided good ideas and directions for the future design.

\section{CORRESPONDING AUTHOR}

Jiaqing Jian jianjia620@126.com 13349897816

\section{REFERENCES}

[1] Li Aihua,Zhang Quoquan, Li Yanling. Research on ceramic title automatic packaging corner machine based on extension theory[J].Applied Mechanics and Materials,2013,420,99-104

[2] G Pahl, W Beitz. Engineering Design. London: Design Council,1984

[3] Frence M J. Conceptual Design for Engineers (Second Edition).London:Springer-verlag,1985

[4] Chen shouyu, Engineering fuzzy set theory and application[M],Beijing: Defense industry Press,1998:5-6

[5] Chen shouyu, unit systematic theory of fuzzy sets decision-making and its application to reallocation of replaced water of the Yellow River[J],Journal of Dalian University of technology, 2006(1):99-102

[6] Chen shouyu, Theory and model of engineering variable fuzzy--Mathematical basis for fuzzy hydrology and water resources[J],Journal of Dalian University of Technology,2005,45, (2)308-312

[7] Chen shouyu, variable fuzzy methods mistake demonstration in extension dependence Function[J],Water resources and power,2005,23(5):1-14

[8] Miao hongbing,Liu zhao, Study on evaluation method of conceptual design scheme of mechanical system[J],Journal of Mechanical Transmission,2008,32(1):37-4 